\title{
Promotion of osteoclast survival and antagonism of bisphosphonate-induced osteoclast apoptosis by glucocorticoids
}

\author{
Robert S. Weinstein, Jin-Ran Chen, Cara C. Powers, Scott A. Stewart, Reid D. Landes, \\ Teresita Bellido, Robert L. Jilka, A. Michael Parfitt, and Stavros C. Manolagas
}

Division of Endocrinology and Metabolism, Center for Osteoporosis and Metabolic Bone Diseases, Department of Internal Medicine, and the Central Arkansas Veterans Healthcare System, University of Arkansas for Medical Sciences, Little Rock, Arkansas, USA

Address correspondence to: Robert S. Weinstein, University of Arkansas for Medical Sciences, Division of Endocrinology and Metabolism, 4301 W. Markham Street, Slot 587, Little Rock, Arkansas 72205-7199, USA. Phone: (501)686-5130; Fax: (501)686-8148; E-mail: weinsteinroberts@uams.edu.

Received for publication October 31, 2001, and accepted in revised form March 11, 2002.

Glucocorticoids depress bone formation by inhibiting osteoblastogenesis and increasing osteoblast apoptosis. However, the role of bone resorption in the initial rapid phase of bone loss characteristic of glucocorticoid-induced osteoporosis is unexplained, and the reason for the efficacy of bisphosphonates in this condition remains unknown. We report that in murine osteoclast cultures, glucocorticoids prolonged the baseline survival of osteoclasts and antagonized bisphosphonate-induced caspase activation and apoptosis by a glucocorticoid receptor-mediated action. Consistent with the in vitro evidence, in a murine model of glucocorticoid-induced osteoporosis, the number of cancellous osteoclasts increased, even though osteoclast progenitor number was reduced. Moreover, in mice receiving both glucocorticoids and bisphosphonates, the expected proapoptotic effect of bisphosphonates on osteoclasts was abrogated, as evidenced by maintenance of osteoclast numbers and, additionally, loss of bone density. In contrast, bisphosphonate administration prevented glucocorticoidinduced osteoblast apoptosis. These results indicate that the early loss of bone with glucocorticoid excess is caused by extension of the life span of pre-existing osteoclasts, an effect not preventable by bisphosphonates. Therefore, the early beneficial effects of these agents must be due, in part, to prolonging the life span of osteoblasts.

J. Clin. Invest. 109:1041-1048 (2002). DOI:10.1172/JCI200214538.

\section{Introduction}

The adverse effects of glucocorticoid excess on the skeleton are marked soon after drug administration is initiated $(1,2)$. Thus, bone mineral density decreases by $2-4.5 \%$ after just 6 months of glucocorticoid administration to healthy men (3), but subsequently, the rate of bone loss declines (4). Considerable evidence indicates that both the early and later phases of bone loss are associated with reduced bone formation $(3,5,6)$, but the role of bone resorption has not been precisely defined, and increased, decreased, or unchanged bone resorption have been reported (5-11). Treatment of the disorder with calcium, vitamin D metabolites, or fluoride has been generally disappointing (11). However, bisphosphonates apparently prevent bone loss (12) and increase bone mass in patients already receiving glucocorticoids (13).

Bisphosphonates are potent analogues of inorganic pyrophosphate that are completely resistant to enzymatic hydrolysis and have a strong affinity for calcium phosphate (14). After binding avidly to bone, bisphosphonates are positioned to be powerful inhibitors of bone resorption due to their uptake by osteoclasts. Some bisphosphonates, such as etidronate and clodronate, can be metabolized to cytotoxic, nonhydrolyzable analogues of ATP. The more potent nitrogen-containing bisphosphonates, such as pamidronate and alendronate, are not metabolized and, instead, inhibit prenylation of small GTP-binding proteins such as Ras (15). Both mechanisms lead to osteoclast apoptosis.

Despite the uncertainty about the role of bone resorption in the pathogenesis of osteoporosis induced by long-term glucocorticoid administration, the benefits of bisphosphonate therapy in this disorder have been ascribed solely to antiresorptive effects and preservation of, or increases in, bone density (13, $16,17)$, but bisphosphonates also have the potential to increase focal bone formation by preventing osteoblast apoptosis $(18,19)$.

We have reported previously that glucocorticoid administration to mice for 27 days (a period representing chronic rather than short-term drug exposure) decreases the number of osteoblast and osteoclast progenitors, decreases the cancellous osteoblast and osteoclast perimeters, and increases osteoblast apoptosis (20). As in mice, decreased osteoblast and osteoclast perimeters, as well as increased osteoblast apoptosis, were documented in patients with glucocorticoid- 
induced osteoporosis $(11,20)$. However, the role of bone resorption in the early phase of glucocorticoidinduced bone loss remains unclear.

We now report that in mice treated with glucocorticoids for 10 days, the number of osteoclasts on cancellous bone increased, despite a decrease in the number of osteoclast progenitors in the bone marrow. In murine osteoclast cultures, glucocorticoids enhanced osteoclast survival, antagonized bisphosphonateinduced activation of caspase- 3 , caspase- 8 , and caspase-9 through a mechanism using the glucocorticoid receptor, and prevented one of the well-known actions of bisphosphonates, the induction of osteoclast apoptosis (14). Consistent with the in vitro evidence, in mice receiving both glucocorticoids and bisphosphonates the expected proapoptotic effect of bisphosphonates on osteoclasts was abrogated, as evidenced by maintenance of cancellous osteoclast numbers and loss of bone density. Despite this early failure to prevent bone loss and decrease osteoclast numbers, bisphosphonate administration did prevent glucocorticoid-induced osteoblast apoptosis.

\section{Methods}

Animals. C57Bl and Swiss Webster mice (Charles River Laboratories, Stone Ridge, New York, USA) were used. At 4 months of age, male Swiss Webster mice were electronically tagged (Biomedic Data System Inc., Maywood, New Jersey, USA) and kept in plastic cages (1 animal per cage) under standard laboratory conditions with a 12-hour dark/12-hour light cycle, a constant temperature of $20^{\circ} \mathrm{C}$, and humidity of $48 \%$. All mice were fed on a standard rodent diet (Agway RMH 3000; Arlington Heights, Illinois, USA) containing $22 \%$ protein, $5 \%$ fat, $5 \%$ fiber, $6 \%$ ash, $3.5 \mathrm{Kcal} / \mathrm{g}, 1.0 \mathrm{IU}$ vitamin $\mathrm{D}_{3} / \mathrm{g}, 0.97 \%$ calcium, and $0.85 \%$ phosphorus with water ad libitum. The animals were weighed at the beginning and end of each experiment. The University of Arkansas for Medical Sciences (UAMS) Division of Laboratory and Animal Medicine approved the protocols.

Osteoclast survival assay. Bone marrow cells were harvested from femora of C57Bl mice (Charles River Laboratories) and cultured in $\alpha$ MEM media with $10 \%$ FBS (HyClone Laboratories, Logan, Utah, USA) for 2 days. Nonadherent cells were collected and aliquots of $0.2 \times 10^{6}$ cells were plated on 16 -well chamber slides in aMEM media with $10 \%$ FBS containing $30 \mathrm{ng} / \mathrm{ml}$ human recombinant M-CSF (R \& D Systems Inc., Minneapolis, Minnesota, USA) and $30 \mathrm{ng} / \mathrm{ml}$ receptor activator of NF- $\mathrm{KB}$ ligand (RANKL) (Amgen Inc., Thousand Oaks, California, USA) for 3-4 days. Media was then changed to fresh $\alpha M E M, M-C S F$, and RANKL with $10 \%$ charcoal-stripped FBS, and the cells were incubated in triplicate for 1 hour with vehicle or $10^{-5} \mathrm{M}$ alendronate before addition of $10^{-7}$ to $10^{-10} \mathrm{M}$ dexamethasone for 24 hours. Floating cells were removed and the attached cells fixed with $2 \%$ paraformaldehyde for 15 minutes at room temperature. Floating cells average about 10 per well and attached cells $200 \pm 40$; thus, it is unlikely that exclusion of the floating cells interfered with the assessment. After washing with TBS, incubating with 3\% hydrogen peroxide for 15 minutes, and blocking with $10 \%$ goat serum for $30-60$ minutes, cells were incubated with active caspase- $3 \mathrm{Ab}$ (Santa Cruz Biotechnology Inc., Santa Cruz, California, USA) (1:50 in $2 \%$ goat serum in TBS) for 2 hours followed by biotinylated anti-rabbit $\mathrm{Ab}$ for 30 minutes and immunoperoxidase staining. Tartrate-resistant acid phosphatase (TRAPase) counterstaining was used to measure total osteoclast number. Apoptotic osteoclasts were identified as dark brown cells (active caspase positive) in each well and expressed as the percentage of total osteoclasts per well.

In addition, cells were incubated in triplicate for 1 hour with vehicle or $10^{-5} \mathrm{M}$ alendronate before addition of $10^{-9} \mathrm{M}$ dexamethasone, $10^{-8} \mathrm{M}$ mifepristone (RU 486), or both for 24 hours, and aliquots of the supernatants from the wells were evaluated using fluorogenic substrates (Biomol Research Laboratories, Plymouth Meeting, Pennsylvania, USA) to measure the enzyme activity of caspase-3, caspase-8, and caspase-9.

Bone densitometry. Dual-energy $\mathrm{x}$-ray absorptiometry (DEXA) was used to determine spinal bone mineral density (BMD) in live mice as previously described (21, 22). Over the past 3 years, the coefficient of variation of the measurement done on a plastic-embedded whole mouse skeleton was $1.8 \%(n=202)$. BMD determinations were done at 2-week intervals to identify the peak adult bone mass of the mice, which was reached between 5 and 6 months of age (20-22). Before the experiment began, BMD measurements were repeated to allocate the animals into groups with equivalent spinal BMD values.

Glucocorticoid administration. Slow-release pellets (Innovative Research of America, Sarasota, Florida, USA) of placebo or $2.1 \mathrm{mg} / \mathrm{kg} /$ day of prednisolone were then implanted for 4,10 , or 27 days, as described previously (20). For dynamic histomorphometric measurements at the 10-day time point, tetracycline $\mathrm{HCl}(30 \mathrm{mg} / \mathrm{kg}$ body weight) was given intraperitoneally 6 and 2 days before sacrifice. At the time of sacrifice, bone marrow aspirates were obtained from the right femur for ex vivo marrow cell cultures, and the left distal femur and lumbar vertebrae (L1-L4) were prepared for histomorphometric analysis.

Detection and quantification of osteoblast and osteoclast progenitors. Femoral marrow cells were obtained as described previously (23). The number of osteoblast progenitors (CFU-OB) in the marrow isolate was determined by culturing cells at $2.0 \times 10^{6}$ cells per $10-\mathrm{cm}^{2}$ well for $25-28$ days with irradiated guinea pig feeder cells in phenol red-free $\alpha$ MEM containing $15 \%$ preselected FBS and 1 $\mathrm{mM}$ ascorbate-2-phosphate. One-half of the medium was replaced every 5 days. Colonies containing osteoblasts were visualized by Von Kossa staining.

$\mathrm{CFU}-\mathrm{OB}$ replication in vitro was determined as described previously (23). One aliquot of cells was used to determine the number of CFU-OB per $10^{6}$ 
marrow cells in the initial isolate, as described above. A second aliquot was used to establish replicate cultures of cells in type I collagen gels at $5 \times 10^{6}$ cells in $1 \mathrm{ml}$ of gel, which were then maintained in the absence or presence of $10 \mathrm{nM}$ prednisolone for 7 days. The cells were then dispersed using bacterial collagenase, and the number of CFU-OB within each gel was determined. To calculate the fold increase in CFU-OB during culture in the collagen gels, the number of CFU-OB obtained per gel (after 7 days of culture) was divided by the number of CFU-OB initially incorporated into the collagen gel.

The number of osteoclast progenitors within the marrow isolate was determined by coculturing 75,000 marrow cells with 8,000 UAMS-32 stromal/osteoblastic cells for 8 days in a $2-\mathrm{cm}^{2}$ well in the presence of $10 \% \mathrm{FBS}$ in phenol red-free $\alpha \mathrm{MEM}$ supplemented with $10 \mathrm{nM} 1,25(\mathrm{OH})_{2} \mathrm{D}_{3}$ to stimulate osteoclast formation, as described previously (24). Replicate cultures $(n=4-6)$ were established from each animal. Osteoclastic cells were enumerated after staining for TRAPase; both mononucleated and multinucleated cells were counted.
Bisphosphonate administration. To examine the impact of an antiresorptive agent on the loss of bone density that accompanies glucocorticoid excess, we pretreated the mice with subcutaneous injections of 0.75 $\mathrm{mg} / \mathrm{kg} /$ day of alendronate (4-amino-1-hydroxybutylidene-1,1-bisphosphonate; obtained from C.W.G.M. Löwik, University Hospital, Leiden, The Netherlands) dissolved in saline or saline alone beginning 3 days before prednisolone or placebo administration and then continued as daily injections during glucocorticoid administration. That this dose of alendronate is adequate has been shown by our previous findings that administration of one-third of this amount of alendronate prevented the increase in urinary free deoxypyridinoline excretion and serum osteocalcin that occurs in mice after ovariectomy (25).

Histomorphometric analysis. The lumbar vertebrae were fixed, embedded undecalcified in methyl methacrylate, and stained as described previously (20-22). The histomorphometric examination was done with a computer and digitizer tablet (OsteoMetrics Inc., Atlanta, Georgia, USA) interfaced to a Zeiss Axioscope (Carl Zeiss Inc., Thornwood, New York, USA) with a drawing
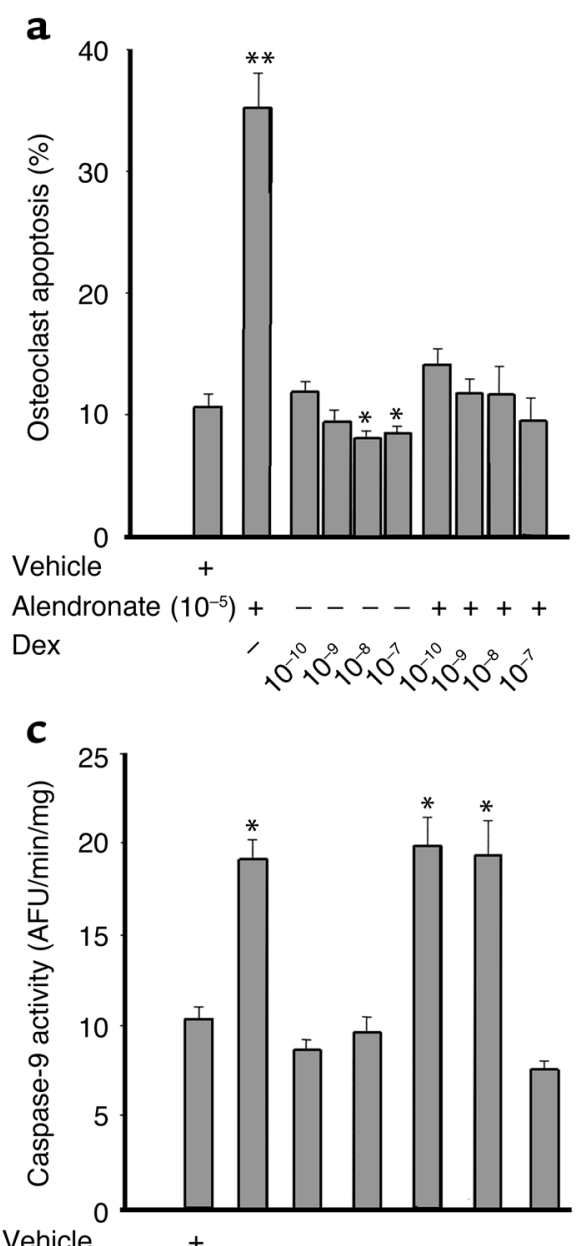

Vehicle

Alendronate $\left(10^{-5}\right)+-+++-$ $\operatorname{Dex}\left(10^{-9}\right) \quad-+++-$ $\mathrm{RU} 486\left(10^{-8}\right) \quad-\quad-\quad-\quad+\quad+$ b

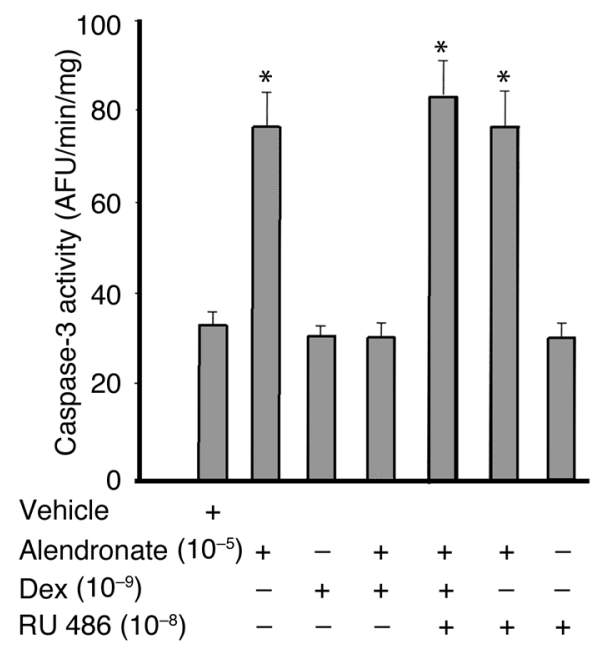

d

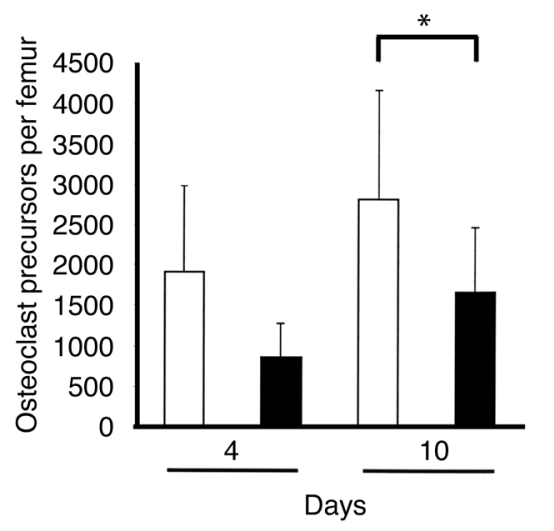

Figure 1

Glucocorticoids alter the birth and death of osteoclasts. (a) Osteoclast apoptosis after vehicle, alendronate, dexamethasone, or preincubation for 1 hour with alendronate followed by dexamethasone. ${ }^{*} P<0.05$ vs. vehicle; ${ }^{*} P<0.0001$ vs. vehicle. (b) Caspase- 3 activation in arbitrary fluorescent units (AFU) per minute per milligram of protein after vehicle; alendronate; dexamethasone; alendronate followed by dexamethasone; alendronate, dexamethasone, and $\mathrm{RU}$ 486; alendronate and RU 486; or RU 486 alone. ${ }^{*} P<0.0001$ vs. vehicle. (c) Caspase-9 activation after vehicle; alendronate; dexamethasone; alendronate followed by dexamethasone; alendronate, dexamethasone and RU 486; alendronate and RU 486; or RU 486 alone. ${ }^{*} P<0.005$ vs. vehicle. (d) Quantification of osteoclast precursors in bone marrow taken from animals sacrificed at 4 and 10 days after exposure to placebo (white bars) or prednisolone (black bars). ${ }^{*} P<0.02$ vs. placebo. 
Table 1

Vertebral bone histomorphometry and BMD in Swiss Webster mice treated with prednisolone and/or alendronate for 10 days.

$\begin{array}{lcccc}\text { Histomorphometric determination } & \begin{array}{c}\text { Placebo and } \\ \text { vehicle }\end{array} & \begin{array}{c}\text { Prednisolone } \\ \text { and vehicle }\end{array} & \begin{array}{c}\text { Placebo and } \\ \text { alendronate }\end{array} & \begin{array}{c}\text { Prednisolone } \\ \text { and alendronate }\end{array} \\ \text { Osteoid area/bone area (\%) } & 0.93 \pm 0.58 & 0.08 \pm 0.04^{\mathrm{A}} & 0.34 \pm 0.10^{\mathrm{B}} & 0.05 \pm 0.02 \\ \text { Osteoid perimeter/bone perimeter }(\%) & 11.27 \pm 5.45 & 1.33 \pm 0.77^{\mathrm{A}} & 5.16 \pm 1.71^{\mathrm{A}} & 0.72 \pm 0.26 \\ \text { Osteoid width }(\mu \mathrm{m}) & 2.00 \pm 0.21 & 1.54 \pm 0.20 & 1.83 \pm 0.21 & 1.91 \pm 0.32 \\ \text { Osteoblast perimeter/bone perimeter }(\%) & 7.31 \pm 3.24 & 0.64 \pm 0.34^{\mathrm{A}} & 2.64 \pm 0.66^{\mathrm{A}} & 0.35 \pm 0.12 \\ \text { Osteoblast number/bone perimeter }(\mathrm{mm}) & 7.85 \pm 3.61 & 0.77 \pm 0.43^{\mathrm{A}} & 3.26 \pm 0.94^{\mathrm{A}} & 0.44 \pm 0.18 \\ \text { Osteoblast apoptosis }(\%) & 5.40 \pm 1.85 & 11.44 \pm 3.91^{\mathrm{A}} & 6.58 \pm 5.26 & 6.99 \pm 1.81^{\mathrm{C}} \\ \text { Osteoclast perimeter/bone perimeter }(\%) & 2.21 \pm 0.60 & 3.30 \pm 1.02 & 1.27 \pm 0.82 & 2.80 \pm 0.94 \\ \text { Osteoclast number/bone perimeter }(\mathrm{mm}) & 1.23 \pm 0.38 & 2.23 \pm 0.54^{\mathrm{B}} & 0.62 \pm 0.27^{\mathrm{B}} & 1.26 \pm 0.13 \\ \text { Reversal perimeter/bone perimeter }(\%) & 20.81 \pm 3.00 & 35.06 \pm 7.75^{\mathrm{B}} & 27.67 \pm 8.66 & 32.65 \pm 14.61 \\ \text { Osteoclast/osteoblast ratio }(\%) & 0.33 \pm 0.09 & 7.00 \pm 5.14^{\mathrm{B}} & 0.54 \pm 0.41 & 8.71 \pm 3.17 \\ \text { Osteoclast/osteoblast ratio }(\mathrm{mm}) & 0.17 \pm 0.04 & 3.79 \pm 2.43^{\mathrm{B}} & 0.22 \pm 0.13 & 3.44 \pm 1.70 \\ \text { Mineralizing perimeter/bone perimeter }(\%) & 5.20 \pm 1.72 & 0.65 \pm 0.11^{\mathrm{A}} & 0.96 \pm 0.51^{\mathrm{A}} & 0.29 \pm 0.13 \\ \text { Mineral appositional rate }(\mu \mathrm{m} / \text { day) } & 1.25 \pm 0.25 & 0.86 \pm 0.21 & 0.99 \pm 0.29 & 1.26 \pm 0.53 \\ \text { Bone formation rate/bone perimeter }\left(\mu \mathrm{m}^{2} / \mu \mathrm{m} / \text { day) }\right. & 0.064 \pm 0.023 & 0.006 \pm 0.002^{\mathrm{A}} & 0.009 \pm 0.005^{\mathrm{A}} & 0.004 \pm 0.001 \\ \text { Spinal BMD }(\% \text { change) } & -1.59 \pm 5.28 & -10.16 \pm 5.24^{\mathrm{B}} & -2.52 \pm 3.00 & -8.93 \pm 2.96^{\mathrm{B}} \\ & & & \end{array}$

Values are expressed as the mean $\pm \mathrm{SD}\left(n=5-9\right.$ animals per group). ${ }^{\mathrm{A} P}<0.002$ and ${ }^{\mathrm{B}} P<0.02$ vs. placebo and vehicle; ${ }^{\mathrm{C}} P<0.05$ vs. prednisone and vehicle; all by ANOVA.

tube attachment. The identity of each specimen was concealed from the histomorphometry reader. All measurements were two-dimensional, confined to the secondary spongiosa, and made at $\times 400$ magnification (numerical aperture 0.75 ). The terminology used was that recommended by the Histomorphometry Nomenclature Committee of the American Society for Bone and Mineral Research (26).

Static measurements of cancellous bone. Cancellous bone area, trabecular width, and osteoid area, perimeter, and width were measured as described previously (20). The osteoblast perimeter was expressed as a percentage of the total cancellous perimeter and also as the number of osteoblasts palisading osteoid per millimeter of cancellous perimeter. Likewise, the osteoclast perimeter was expressed as a percentage of the total cancellous perimeter covered by TRAPase-positive osteoclasts and as the number of osteoclasts per millimeter of cancellous perimeter. The ratio of osteoclasts to osteoblasts was also expressed as both the percentage and number of cells.

Dynamic measurements of cancellous bone. The rate of mineral apposition was calculated as the mean distance between the midpoints of the two tetracycline labels divided by the interdose duration (4 days). The mineralizing perimeter and rate of bone formation per cancellous perimeter (square micrometer per micrometer per day) were calculated as described previously $(20,21)$.

Measurement of apoptosis in undecalcified bone sections. Apoptosis was detected by in situ nick-end labeling (ISEL) using Klenow terminal deoxynucleotidyl transferase (Oncogene Research Products, Cambridge, Massachusetts, USA) as described previously (27). Sections were counterstained with $0.5-3 \%$ methyl green. Plastic-embedded sections of vertebrae taken from orchidectomized adult mice were used as a positive control. Omitting the transferase made negative controls. Apoptotic osteoblasts were identified as ISEL-positive cells lining the osteoid-covered cancellous perimeter.

Statistics. In the osteoclast apoptosis assay, drug effects were examined using one-way ANOVA. In addition, a dose-response test for a linear trend between vehicle and dexamethasone was done using linear contrast coefficients. To evaluate the changes in ex vivo marrow cell cultures and 4- and 10-day BMD measurements, we used two-way ANOVA. If variances were unequal, a Satterthwaite approximation was used to determine the degrees of freedom. Differences between group means with the CFU-OB replication assay were evaluated with Student $t$ tests. Serial changes in BMD were analyzed using a mixed effects model of repeated measures (28). Histomorphometric data were examined by one-way ANOVA. Comparisons of interest were specified a priori and their $P$ values adjusted with Bonferroni's correction (29). Pearson correlation coefficients were calculated to test for an association between two independently measured variables. $P$ values less than 0.05 were considered significant.

\section{Results}

Effects of glucocorticoids on osteoclast survival in vitro. As expected, addition of alendronate $\left(10^{-5} \mathrm{M}\right)$ to osteoclast cultures obtained from murine bone marrow cells in the presence of M-CSF and RANKL caused more than a threefold increase in the percentage of apoptotic osteoclasts (Figure 1a). Strikingly, when dexamethasone at $10^{-10}$ to $10^{-7} \mathrm{M}$ was combined with the alendronate, osteoclast apoptosis was completely abrogated, with values not significantly different from that seen with vehicle alone. Dexamethasone alone also reduced osteoclast apoptosis with the $10^{-8}$ and $10^{-7} \mathrm{M}$ concentrations as compared with vehicle. Furthermore, there was a linear trend for the doses of dexamethasone 

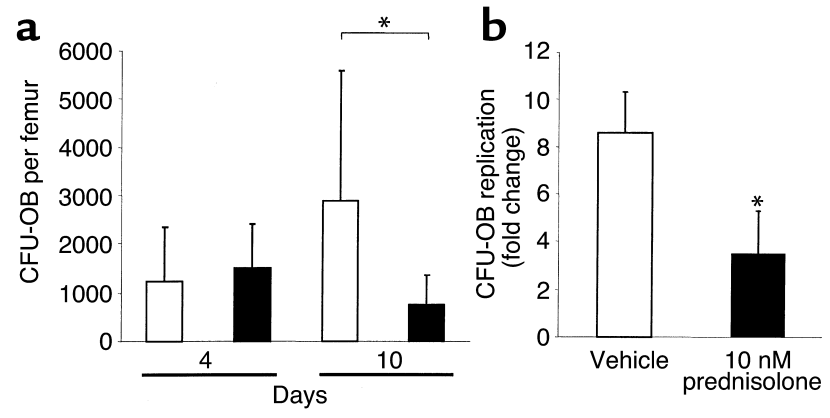

Figure 2

(a) Quantification of CFU-OB formed in bone marrow taken from animals sacrificed at 4 and 10 days after exposure to placebo (white bars) or prednisolone (black bars). Marrow cells were obtained from the femurs of male mice, and cells from each mouse were cultured separately. Each bar represents the mean $( \pm S D)$ values from 7-10 animals. ${ }^{*} P<0.03$ vs. placebo. (b) Results from the in vitro osteoblast replication assay using vehicle (white bar) or $10 \mathrm{nM}$ prednisolone (black bar). Determinations were done in triplicate. ${ }^{*} P<0.05$ vs. placebo.

alone to reduce the percentage of apoptotic osteoclasts as compared with vehicle $(P<0.0002)$, strong evidence that glucocorticoids increase osteoclast survival even in the absence of an added proapoptotic agent. Similar results were seen in a second experiment.

Consistent with the results above, dexamethasone completely inhibited alendronate-induced activation of caspase-3 (Figure 1b) and caspase-9 (Figure 1c). Dexamethasone also inhibited a 2.4-fold increase in caspase- 8 activation induced by alendronate $(P<0.02$, data not shown). This effect was evidently mediated by the glucocorticoid receptor as revealed by its full blockade with $10^{-8} \mathrm{M}$ RU 486, a potent glucocorticoid receptor antagonist (30). RU 486 had no effect of its own on activation of caspase-3, caspase-8, or caspase-9. Similar results were reproduced in a second experiment.

Effects of glucocorticoid administration on bone marrow content of osteoclast and osteoblast progenitors. In ex vivo marrow cell cultures from mice receiving prednisolone for 4 or 10 days, the number of osteoclastic cells formed in response to $1,25(\mathrm{OH})_{2} \mathrm{D}_{3}$ was decreased by $55 \%$ and $41 \%$, respectively (Figure 1d), a finding reflecting a reduction in the number of osteoclast progenitors in the bone marrow.

In parallel bone marrow cell cultures from the animals receiving prednisolone, the number of osteoblast progenitors represented by $\mathrm{CFU}-\mathrm{OB}$ colonies decreased by $74 \%$ after 10 days of glucocorticoid administration when compared with placebo controls, but there was no significant change after only 4 days (Figure 2a). Consistent with the prednisolone-induced reduction in CFU-OB, $10 \mathrm{nM}$ prednisolone in vitro attenuated the replication of these transit-amplifying osteoblast progenitors in murine bone marrow cells by approximately $60 \%$ (Figure $2 b$ ).

Demonstration of early loss of bone density in mice receiving prednisolone. In an experiment that lasted 27 days, animals receiving prednisolone $(n=7)$ exhibited signifi- cant early decreases in spinal BMD from that seen in the placebo group $(n=8)$ by 6 days after drug administration (7.4\%) (Figure 3a). This was followed by an additional $2 \%$ decrease from the day 6 values by day 27 , a two-phase time course similar to that found in patients treated with glucocorticoids. In the placebo group of these 7-month-old animals, the decrease in BMD was not significant. The prednisolone-induced early loss of BMD has been reproduced in multiple experiments $[n=6 ; 8.8 \% \pm 1.0(\mathrm{SEM})]$. The bone loss was not due to changes in food intake, body weight, or seminal vesicle weight, and hepatic fatty infiltration was absent, consistent with previous observations (20). In a second experiment, we sacrificed animals at 4 and 10 days to bracket the 6-day time point and, in the case of the latter time point, to allow sufficient time to administer two doses of tetracycline by day 10 for measurement of the bone formation rate. Mice implanted with prednisolone pellets (7-10 animals per group) exhibited a $10 \%$ decline in spinal BMD when compared with animals receiving placebo pellets after 10 days of glucocorticoid administration. However, there were no significant changes in BMD after only 4 days (Figure 3b).

Effects of glucocorticoid administration for 10 days on vertebral bone histomorphometry. With prednisolone administration, the number of osteoclasts per millimeter of the cancellous perimeter was increased by $81 \%$, resulting in a greater than 20 -fold increase in the ratio of cancellous osteoclasts to osteoblasts
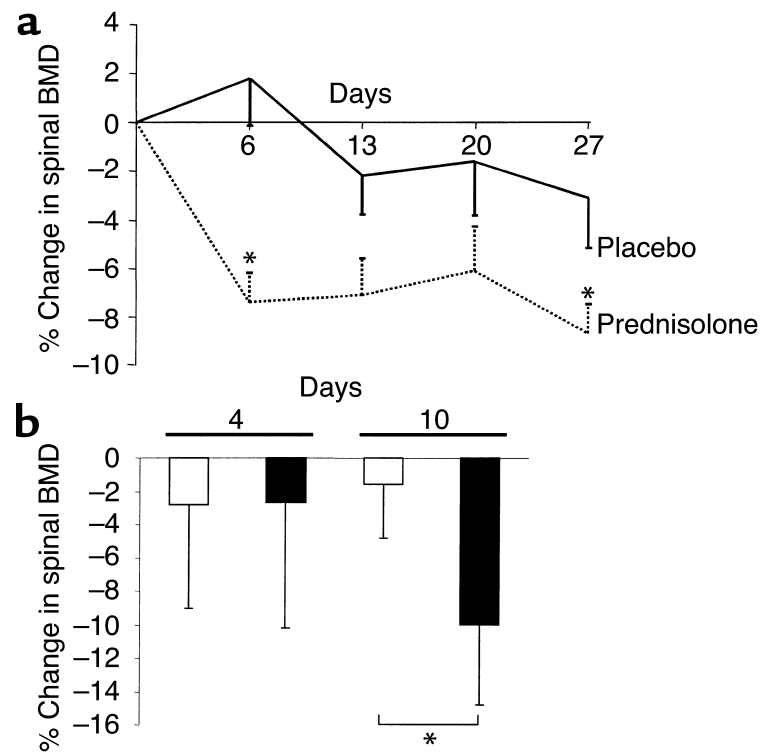

Figure 3

Bone density in mice receiving prednisolone. (a) Serial measurements of the percentage of change in murine spinal BMD determinations (by DEXA) in animals receiving placebo (solid line) or prednisolone (dotted line) are plotted against time in days. Each point represents the mean values ( \pm SEM) of 7-8 animals. ${ }^{*} P<0.05$ from placebo by a mixed-effects model of repeated measures. (b) Quantification of the changes in murine spinal BMD in vivo at 4 and 10 days. Each bar represents the mean $( \pm S D)$ values from 7-10 animals receiving placebo (white bars) or prednisolone (black bars). ${ }^{*} P<0.02$ vs. placebo. 


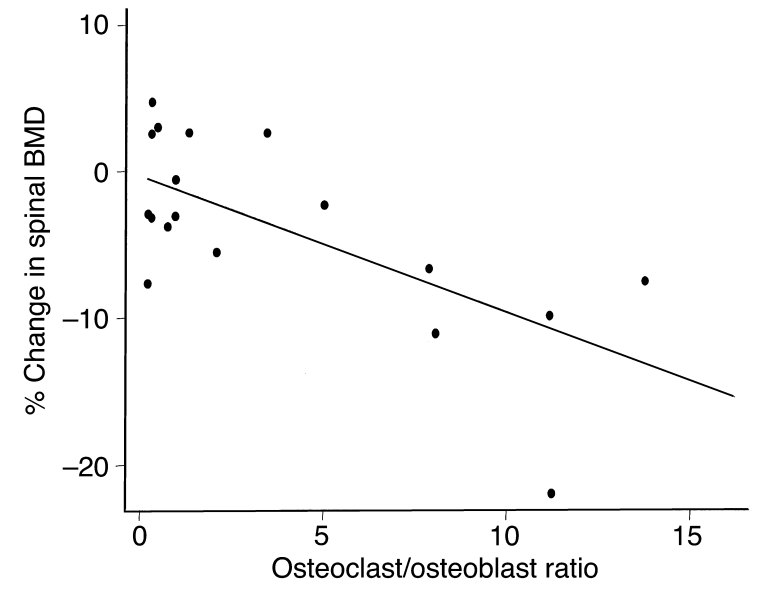

Figure 4

The percentage of change in spinal BMD in Swiss Webster mice is plotted against the cancellous osteoblast/osteoclast ratio on a percentage of bone perimeter referent $(r=-0.73, P<0.01)$.

(Table 1). Erosion cavities devoid of osteoclasts, the reversal perimeter, were also increased by $68 \%$, a finding reflecting delayed bone formation.

Lumbar cancellous bone demonstrated a 91\% decrease in the rate of bone formation. Consistent with the fall in bone formation, osteoid area, osteoid perimeter, osteoblast perimeter, and mineralizing perimeter significantly decreased by $88-91 \%$, but the reduction in osteoid width was not significant. The change in spinal BMD inversely correlated with the cancellous osteoclast-to-osteoblast ratio on a percentage of bone perimeter referent (Figure 4). In this short-term experiment, there were no significant changes in cancellous bone area or trabecular width (data not shown).

As reported previously by Hughes et al., the prevalence of cancellous osteoclast apoptosis in 4-week-old mice is only $0.2 \%$ (31). In 7 -month-old mice, we were able to detect only $1.23 \pm 0.38$ osteoclasts $/ \mathrm{mm}$ (Table 1) or about $66 \pm 14$ osteoclasts per cancellous bone longitudinal section of L1-4. Using the above numbers, $0.13 \pm 0.03$ apoptotic osteoclasts could have been present. Because of this scarcity, we were unable to determine the prevalence of this phenomenon.

In vertebrae taken from mice receiving prednisolone, there was an $112 \%$ increase in cancellous osteoblast apoptosis when compared with controls (Table 1). Morphological changes typical of apoptosis accompanied the ISEL-positive osteoblasts and included discretely condensed chromatin, nuclear fragmentation, and cell shrinkage (Figure 5).

Effects of bisphosphonate administration on prednisoloneinduced bone changes. In animals receiving alendronate and placebo pellets, the expected decrease in bone remodeling (32) was manifest as diminished osteoid area and perimeter, decreased osteoblast perimeter, and reductions in mineralizing perimeter and bone formation rate (Table 1). Consistent with the unimpaired mineralization with alendronate administration observed previously (33), osteoid width remained normal. Moreover, the number of osteoclasts per millimeter of cancellous bone decreased 50\% when compared with animals receiving vehicle and placebo pellets. Thus, alendronate bioavailability was adequate. However, even when given as daily injections for 3 days before and continued throughout prednisolone administration, alendronate partially attenuated but did not stop the significant decline in spinal BMD measured at 10 days and failed to decrease the number of osteoclasts on cancellous bone; it did, however, prevent glucocorticoid-induced apoptosis of cancellous osteoblasts (Table 1). Maintenance of cancellous osteoclasts in the prednisolone and alendronate group is particularly conspicuous considering the concurrent $56 \%$ decrease in the rate of bone formation.

\section{Discussion}

Skeletal maintenance during normal bone remodeling requires balance between the number of mature osteoclasts and osteoblasts that implement the remodeling process. Such balance is determined by the frequency of division of the appropriate precursors and the life span of their progeny, reflecting the timing of death by apoptosis (34). The results of the present short-term studies demonstrate that 10 days of glucocorticoid administration increases osteoclast numbers despite decreased osteoclast production, most likely by decreasing osteoclast apoptosis. This effect is temporally linked with loss of BMD. Moreover, our results indicate that glucocorticoids antagonize the proapoptotic effect of bisphosphonates on osteoclasts. The mechanism underlying this antagonism involves inhibition of alendronate-induced activation of caspase-3, the major effector caspase activated in osteoclasts undergoing apoptosis following exposure to nitrogencontaining bisphosphonates (35). In addition, we have shown that alendronate stimulates activation of caspase- 8 and caspase- 9 and that this activation is also

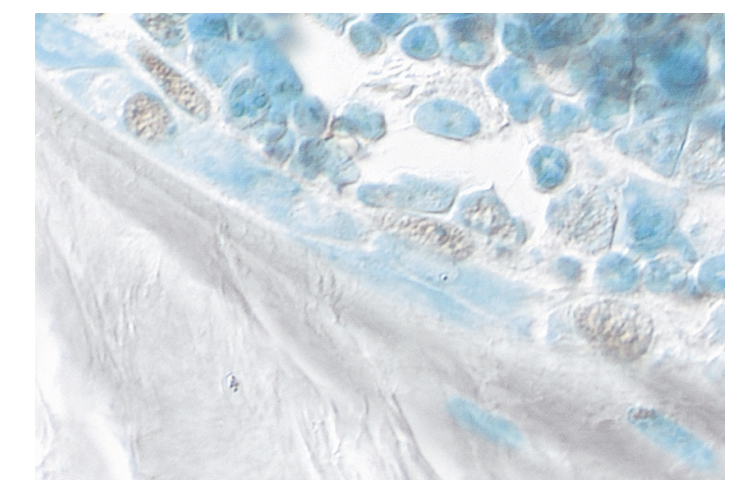

\section{Figure 5}

Osteoblast apoptosis in murine vertebral cancellous bone. Apoptotic osteoblasts were identified by brown ISEL staining and morphological features such as nuclear fragmentation and chromatic condensation. Methyl green counterstain viewed with Nomarski differential interference microscopy (original magnification $\times 630$ ). 
antagonized by dexamethasone. Furthermore, the inhibitory effect of dexamethasone is mediated by the glucocorticoid receptor as revealed by full blockade of the inhibition with RU 486.

At any time during the excavation of an erosion bay, the number of resident osteoclasts depends both on the number initially assembled on the activated bone surface and on the number that have so far escaped death by apoptosis. Each osteoclast has only a temporary existence, and continuing bone resorption requires the uninterrupted arrival of new preosteoclasts from the circulation (36). Based on dynamic histomorphometry of the murine vertebral secondary spongiosa, we calculated that the mean active life span of an osteoblast on cancellous bone is about 12 days (20). In humans, the ratio of the life span of an osteoclast to that of an osteoblast is 21 days/90 days (36). From these calculations, we estimate that the mean active life span of a murine osteoclast is about 3 days. This estimate is consistent with our conclusion that an increase in the number of cancellous osteoclasts after 10 days of glucocorticoid administration, despite a reduction in osteoclastogenesis, could only result from prolongation of osteoclast life span. Our estimate may have a higher margin of error than would be ideal, but the increase in cancellous osteoclast numbers in spite of decreased production of osteoclast progenitors after 10 days of glucocorticoid administration strongly indicates an increase in osteoclast life span. Our observations are also consistent with our earlier findings of a doubling of the osteoclast perimeter by day 7 of glucocorticoid administration (20). By day 10, these events, although beginning to wane, still result in a greater than 20 fold increase in the ratio of osteoclasts to osteoblasts and in loss of BMD (Figure 4).

We have found previously that administration of alendronate with prednisolone prevented loss of BMD after 8 weeks (18). In the present experiment, 10 days of alendronate did not prevent glucocorticoid-induced loss of BMD or decrease the number of cancellous osteoclasts, but it did protect osteoblasts from glucocorticoid-induced apoptosis. These findings are consistent with the evidence obtained in the rat that even though hydrocortisone and dexamethasone inhibit osteoclast recruitment, they stimulate bone resorption by existing osteoclastic cells (37). Furthermore, corticosterone has been shown to cause a transient increase in osteoclast number and bone resorption in cultures of fetal rat parietal bones (38).

If the initial increase in bone resorption is due to prolongation of osteoclast life span, which is refractory to bisphosphonates, how do these agents prevent bone loss and reduce bone turnover in glucocorticoid-treated patients? In most clinical studies, the earliest measurements are made at 3-6 months after initiation of treatment (11-13), so that very early bone loss could have escaped detection. Nevertheless, the mechanism of the bone protection requires explanation. The impact of the antiapoptotic effect of glucocorticoids on osteoclasts might wane with time because of the fall in osteoclastogenesis. Eventually the proapoptotic effect of bisphosphonates may become more important. According to this scenario, bone turnover is already reduced in glucocorticoid-treated patients, but further reduction by bisphosphonates would contribute to the bone-sparing effect $(11,13)$. However, we propose that the most important effect is the prolongation of osteoblast life span by bisphosphonates, so that even if total body bone formation remained depressed, focal bone formation relative to resorption could be increased.

In conclusion, the demonstration that glucocorticoids prevent osteoclast apoptosis in vitro and in vivo and that this protection cannot be thwarted by bisphosphonate treatment explains the early increase in bone resorption. Glucocorticoids increase osteoclast life span and bisphosphonates decrease it; in the short-term, the glucocorticoid effect outweighs that of the bisphosphonate, but in the long term the balance may be reversed as the decimated osteoclastogenesis fails to supply new osteoclasts. The long-term beneficial effects of bisphosphonates on bone mass may be at least partly due to postponing apoptosis and thus prolonging osteoblast life span.

\section{Acknowledgments}

We thank Charles Vo, Tony Chambers, Julie Crawford, Randal Shelton, and Paula Roberson for their valuable assistance. This material is based upon work supported by a Veterans Affairs Research Enhancement Award Program (REAP) Grant and Veterans Affairs Merit Review Grants (to R.S. Weinstein, R.L. Jilka, and S.C. Manolagas) from the Office of Research and Development, Department of Veterans Affairs, and the NIH (PO1-AG13918 and RO1-AR46191).

1. Fitzpatrick, L.A. 1994. Glucocorticoid-induced osteoporosis. In Osteoporosis. R. Marcus, editor. Blackwell Scientific Publications. Boston, Massachusetts, USA. 202-226.

2. Reid, I.R. 1989. Pathogenesis and treatment of steroid osteoporosis. Clin. Endocrinol. 30:83-103.

3. Pearce, G., Tabensky, D.A., Delmas, P.D., Baker, H.W.G., and Seeman, E. 1998. Corticosteroid-induced bone loss in men. J. Clin. Endocrinol. Metab. 83:801-806.

4. LoCascio, V., et al. 1990. Bone loss in response to long-term glucocorticoid therapy. Bone Miner. 8:39-51.

5. Dempster, D.W., Arlot, M.A., and Meunier, P.J. 1983. Mean wall thickness and formation periods of trabecular bone packets in corticosteroidinduced osteoporosis. Calcif. Tissue Int. 35:410-417.

6. Dempster, D. 1989. A perspective: bone histomorphometry in glucocorticoid-induced osteoporosis. J. Bone Miner. Res. 4:137-141.

7. Stellon, A.J., Webb, A., and Compston, J.E. 1988. Bone histomorphometry and structure in corticosteroid treated chronic active hepatitis. Gut. 29:378-384.

8. Hall, G.M., Spector, T.D., and Delmas, P.D. 1995. Markers of bone metabolism in postmenopausal women with rheumatoid arthritis. Arthritis Rheum. 38:902-906.

9. Lindgren, J.U., Johnell, O., and DeLuca, H.F. 1983. Studies of tissue in rats treated by prednisolone and $1,25(\mathrm{OH})_{2} \mathrm{D}_{3}$. Clin. Orthop. 181:264-268.

10. Carbonare, D.L., et al. 2001. Comparison of trabecular bone architecture and remodeling in glucocorticoid-induced and postmenopausal osteoporosis. J. Bone Miner. Res. 16:97-103.

11. Weinstein, R.S. 2001. Glucocorticoid-induced osteoporosis. Rev. Endocr. Metab. Disord. 2:65-73.

12. Reid, I.R., King, A.R., Alexander, C.J., and Ibbertson, H.K. 1988. Prevention 
of steroid-induced osteoporosis with (3-amino-1-hydroxypropylidene)1,1-bisphosphonate (APD). Lancet. 1:143-146.

13. Saag, K.G., et al. 1998. Alendronate for the prevention and treatment of glucocorticoid-induced osteoporosis. N. Engl. J. Med. 339:292-299.

14. Fleisch, H. 2000. Bisphosphonates in bone diseases: from the laboratory to the patient. Academic Press. San Diego, California, USA. 27-55.

15. Luckman, S.P., et al. 1998. Nitrogen-containing bisphosphonates inhibit the mevalonate pathway and prevent post-translational prenylation of GTP-binding proteins, including Ras. J. Bone Miner. Res. 13:581-589.

16. Boutsen, Y., Jamart, J., Esselinckx, W., and Devogelaer, J.P. 2001. Primary prevention of glucocorticoid-induced osteoporosis with intravenous pamidronate and calcium: a prospective controlled 1-year study comparing a single infusion, an infusion given once every 3 months, and calcium alone. J. Bone Miner. Res. 16:104-112.

17. Reid, D.M., et al. 2000. Efficacy and safety of daily risedronate in the treatment of corticosteroid-induced osteoporosis in men and women: a randomized trial. J. Bone Miner. Res. 15:1006-1013.

18. Plotkin, L.I., et al. 1999. Prevention of osteocyte and osteoblast apoptosis by bisphosphonates and calcitonin. J. Clin. Invest. 104:1363-1374.

19. Weinstein, R.S., and Manolagas, S.C. 2000. Apoptosis and osteoporosis. Am. J. Med. 108:153-164.

20. Weinstein, R.S., Jilka, R.L., Parfitt, A.M., and Manolagas, S.C. 1998. Inhibition of osteoblastogenesis and promotion of apoptosis of osteoblasts and osteocytes by glucocorticoids: potential mechanisms of the deleterious effects on bone. J. Clin. Invest. 102:274-282.

21. Jilka, R.L., Weinstein, R.S., Takahashi, K., Parfitt, A.M., and Manolagas, S.C. 1996. Linkage of decreased bone mass with impaired osteoblastogenesis in a murine model of accelerated senescence. J. Clin. Invest. 97:1732-1740.

22. Weinstein, R.S., Jilka, R.L., Parfitt, A.M., and Manolagas, S.C. 1997. The effects of androgen deficiency on murine bone remodeling and bone mineral density are mediated via cells of the osteoblastic lineage. Endocrinol. 138:4013-4021.

23. Di Gregorio, G.B., et al. 2001. Attenuation of the self-renewal of transient-amplifying osteoblast progenitors in the murine bone marrow by $17 \beta$ estradiol. J. Clin. Invest. 107:803-812.

24. O’Brien, C.A., Gubrij, I., Lin, S-C., Saylor, R.L., and Manolagas, S.C. 1999. STAT3 activation in stromal/osteoblastic cells is required for induction of the receptor activator of NF- $\mathrm{KB}$ ligand and stimulation of osteoclastogenesis by gp130-utilizing cytokines or interleukin- 1 but not 1,25 - dihydroxyvitamin $\mathrm{D}_{3}$ or parathyroid hormone. J. Biol. Chem 274:19301-19308

25. Jilka, R.J., et al. 1998. Loss of estrogen upregulates osteoblastogenesis in the murine bone marrow: evidence for autonomy from factors released during bone resorption. J. Clin. Invest. 101:1942-1950.

26. Parfitt, A.M., et al. 1987. Bone histomorphometry: standardization of nomenclature, symbols, and units. Report of the ASBMR Histomorphometry Nomenclature Committee. J. Bone Miner. Res. 2:595-610.

27. Borton, A.J., Frederick, J.P., Datto, M.B., Wang, X-F, and Weinstein, R.S. 2001. The loss of Smad3 results in a lower rate of bone formation and osteopenia through dysregulation of osteoblast differentiation and apoptosis. J. Bone Miner. Res. 16:1754-1764.

28. Jennrich, R.I., and Schluchter, M.D. 1986. Unbalanced repeated-measures models with structured covariance matrices. Biometrics. 42:805-820.

29. StatCorp. 2001. Stata Statistical Software Release 7.0. Stata Corporation. College Station, Texas, USA. 3:44-115.

30. Spitz, I.M., and Bardin, C.W. 1993. Mifepristone (RU 486)-a modulator of progestin and glucocorticoid action. N. Engl. J. Med. 329:404-412.

31. Hughes, D.E., et al. 1996. Estrogen promotes apoptosis of murine osteoclasts mediated by TGF- $\beta$. Nature Med. 2:1132-1136.

32. Siris, E., et al. 1996. Comparative study of alendronate vs. etidronate for the treatment of Paget's disease of bone. J. Clin. Endocrinol. Metab. 81:961-967.

33. Bone, H.G., et al. 1997. Dose-response relationships for alendronate treatment in osteoporotic elderly women. J. Clin. Endocrinol. Metab. 82:265-274.

34. Manolagas, S.C. 2000. Birth and death of bone cells: basic regulatory mechanisms and implications for the pathogenesis and treatment of osteoporosis. Endo. Rev. 21:115-137.

35. Benford, H.L., McGowan, N.W.A., Helfrich, M.H., Nuttall, M.E., and Rogers, M.J. 2001. Visualization of bisphosphonate-induced caspase-3 activity in apoptotic osteoclasts in vitro. Bone. 28:465-473.

36. Parfitt, A.M. 1998. Osteoclast precursors as leukocytes: importance of the area code. Bone. 23:491-495.

37. Defranco, D.J., Lian, J.B., and Glowacki, J. 1992. Differential effects of glucocorticoid on recruitment and activity of osteoclasts induced by normal and osteocalcin-deficient bone implanted in rats. Endocrinology. 131:114-121.

38. Gronowicz, G., McCarthy, M.B., and Raisz, L.G. 1990. Glucocorticoids stimulate resorption in fetal rat partial bones in vitro. J. Bone Miner. Res. 5:1223-1230. 